\title{
CURRENT ISSUES IN HIGH POWER MICROWAVES
}

\author{
FORREST J. AGEE \\ PHILLIPS LABORATORY \\ 3550 ABERDEEN AVE., S.E. \\ KIRTLAND AFB, NM 87117 \\ (505) 846-2824
}

\begin{abstract}
Technology development in the field of high power microwaves is focused principally in three areas, narrowband sources, ultrawideband sources, and antennas. This paper reviews the status of activity in each area and discusses challenges for power modulators and pulsers that derive from the work presently in progress. Basic research efforts are presently attempting to overcome barriers to further progress in the use of both narrowband and ultra-wideband technologies. Several classes of narrowband tubes are discussed and the course of present basic research efforts that bear upon them is summarized. Success in advancing the art in the areas discussed has the potential for introducing new commercial and military products for applications as diverse as advanced radars, welding, and waste treatment.

\section{High Power Microwaves: Two Fields of Technology}

The field of high power microwave technology is now developing along several lines of research. These offer different possible outcomes in terms of possible uses of the technology and in technical terms of the RF environments that will result. These generally are narrowband RF sources and ultra-wideband emitters and their associated antennas. Narrowband sources are designed to provide high power pulses over a relatively narrow range of frequencies, usually a single frequency. These sources have been used in traditional applications including radars, microwave ovens, materials curing, particle accelerators, and similar applications for which a well defined single frequency works well for the application. Ultra-wide band sources by contrast feature fast rising pulse waveforms, typically less than a nanosecond, and short pulse widths of the order of a few nanoseconds. The pulse repetition frequency can vary widely from a single pulse to
\end{abstract}

tens of kilohertz. These scurces have a broad frequency content by virtue of the pulse parameters, and therefore find application in ultra-wideband synthetic aperture radars and other applications for which a broad spectrum of RF energy is useful.

\section{Narrowband High Power Microwaves}

Many of the narrowband efforts to date have sought to take advantage of the energy available in pulsed power systems to make powerful relativistic electron beams and to use these to drive microwave tubes. They use a variety of mechanisms to convert the electron beam energy to microwave energy. A number of tubes using this approach have succeeded in producing power in excess of a gigawatt in a variety of tubes (reflex diode, relativistic klystron amplifier, relativistic klystron oscillator, and magnetically insulated line oscillator). ${ }^{1,2,3,4}$ The common experience of these accomplishments and those of others operating in excess of 200 $\mathrm{kW}^{5,6}$ is that at very high power operation, three orders of magnitude greater than high power tubes for radars, the microwave production occurs for only short pulses, often much less than the pulse length of the available pulsed power. Typically, the duration is less than 100 ns for power in excess of ${ }^{-1} \mathrm{GW}$.

This problem is ore of intense interest at the present time, and there is a substantial effort underway to achieve a better understanding of the causes of pulse shortening for various types of high power microwave tubes. ${ }^{7}$ There are many possible causes that can be expected to affect some tube types and not others. It has been variously described as being a problem in a hypothesis rich environment and one without a serial killer. ${ }^{8}$ For some tubes, the problem could be caused by unwanted plasma generation arising from stray electrons, by output window breakdown, by cathode effects, by breakdown at sharp edges, etc. There is no reason to expect that the same mechanism will dominate all types 
of tubes, although some may have common problems.

At Phillips Laboratory, recent work on the injection locked relativistic klystron oscillator (RKO) has led to an ability to stretch the RF pulse to the limits of the available pulsed power system by concentrating effort on two areas. The first was to eliminate by removing some sharp edges that coincided with observed emitted light. After this was done, the focus shifted to a careful study of the performance of the cathode. It was determined that the RF production occurred only for a rather precise range of current for a given voltage, and that the performance of the tube was critically dependent upon getting the voltage up quickly. A slowly rising current pulse meant that the tube was only in the right parameter space for microwave production for a fraction of the applied voltage pulse. For about $500 \mathrm{kV}$, the tube only would produce $\mathrm{RF}$ in a range of $+/-5 \%$ at about $9 \mathrm{kA}$. The RKO (Figure 1) has much potential for a number of applications, but it imposes strict requirements upon the modulator used to power it and upon the performance of the cathode. ${ }^{3,9}$

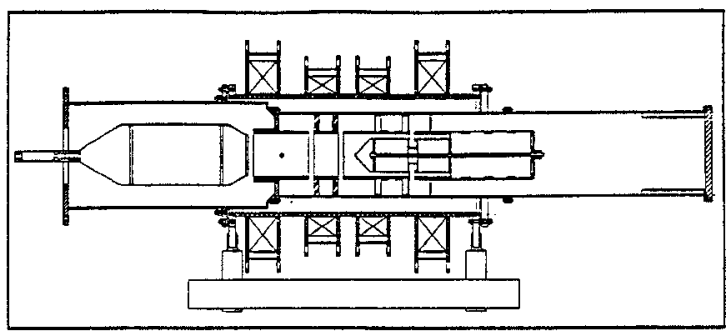

Figure 1. Schematic Diagram of the Relativistic Klystron Oscillator (RKO).

By sharp contrast, the magnetically insulated line oscillator (MILO) has been observed to operate at a constant frequency over a wide range of operating voltage, varying in frequency by less than $10 \mathrm{MHz}$ for a $1.2 \mathrm{GHz}$ tube over a voltage range of $150-500 \mathrm{kV}$. This tube (Figure 2) is similar to the concept of a linear magnetron, with the magnetic field provided by the self field of the relativistic electron beam. The electron beam is launched from an explosive emission cathode, usually of velvet, which is arranged to go through out the region of the vanes that make up the RF choke, slow wave structure, and extraction cavity. This tube operates at low impedance relative to the RKO, of the order 5 to $10 \mathrm{ohms}$. The MILO has been driven by the Phillips Laboratory Rep-rate
Pulser that features a flexible arrangement of elements that allows the pulser to deliver high power (20 GW pulses) with a repetition rate of up to $40 \mathrm{~Hz}$. ${ }^{9,10}$ The RF output power varies as the square of the applied voltage and the tube operation is quite reliable and stable over a wide voltage range (Figure 3 ). ${ }^{11}$

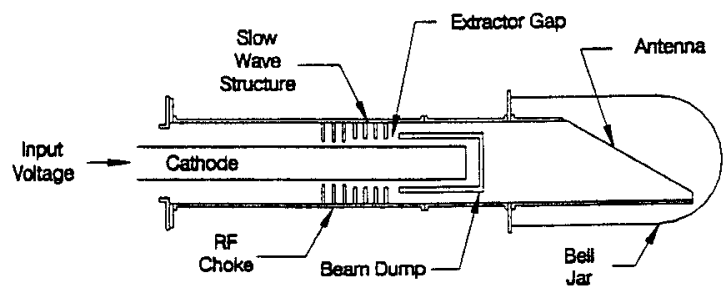

Figure 2. Schematic Diagram of the Magnetically Insulated Line Oscillator MILO).

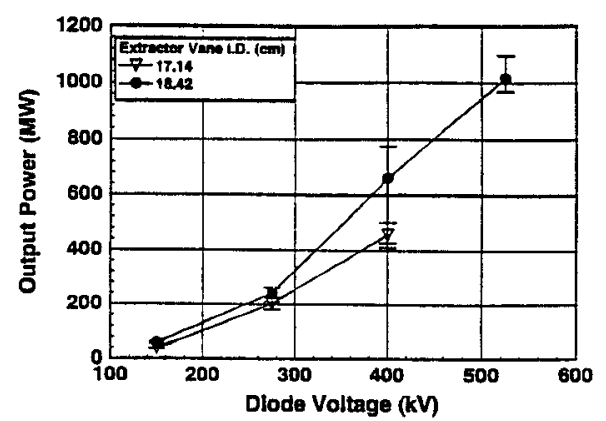

Figure 3. Variation in Output Power with Applied Voltage for MILO. Note wide range of pulsed power performance for which tube operates.

The projected requirements for modulators to drive HPM tubes are summarized in Figure 4., which represents one laboratory's view of what will be needed to support planned research efforts near term. For the longer term, one must make certain assumptions about the future success of the research efforts in the area of pulse shortening to arrive at a commercial position. If the pulse length can be extended to the range of 2 to 10 microseconds, then all sorts of exciting accelerator possibilities may develop. Medical LINACS, for example typically have RF driver pulse lengths of $5-6$ microseconds. The ability to have the RF pulse stay on long enough to fill accelerator cavities would enable significant reductions in the number of tubes required for large and compact accelerators alike. The big research machines could take on a dramatically different form with significant economic impact on the feasibility of large projects. At the small end, very powerful 
compact machines could make deep beam welding for heavy industry very attractive. In this case, the needs would be bracketed by the RKO and the MILO, except that pulse lengths would grow to several microseconds or more in pulse length. For military and civilian applications alike, the size, weight and efficiency of the pulsed power systems will require careful thought and design to fit tight constraints in each category. Typical sizes are 15 to 30 inches in diameter and ten feet in length, with a half ton or so upper limit on weight for some applications.

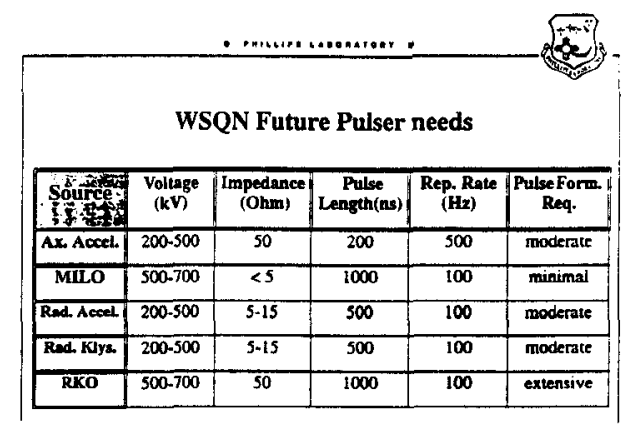

Figure 4. Summary of Projected Pulsed Power Modulator Needs for Experiments Planned at Phillips Laboratory.

\section{Ultra-wide Band (UWB) Source Technology}

The art of UWB technology is aimed in a number of directions today. Broadly, these can be characterized in three groups. The first is the straightforward application of very high voltages to single switches to discharge energy onto a single antenna. The second uses a pulser to feed a carefully designed non-dispersive high gain antenna system to achieve high field strength in the far field of the antenna, and the third approach uses many radiating elements switched at relatively low voltage and low jitter to collectively deliver an additive field at the target of the array. The Phillips Laboratory is working on all three concepts, with encouraging results for each. ${ }^{12}$

For the first approach, pulsers with an operating voltage of up to $3 \mathrm{MV}$ and repetition rates of 1 to $10 \mathrm{kHz}$ are interesting. At present, we are building at the $1 \mathrm{MV}$ level and designing for $2 \mathrm{MV}$ in the high pressure hydrogen gas Hindenberg series of pulsers. The $\mathrm{H}-3$ system that is the present state of the art, with an operating voltage of $1 \mathrm{MV}$, peak output power of $47 \mathrm{GW}$, and a repetition rate of $2 \mathrm{kHz}$. This technology is one that demands much in getting the energy radiated efficiently through a single antenna. The parameters exceed the knowledge base for fast transients on materials, and this is an area of study by university and government laboratories; under AFOSR sponsorship.' The pulser relies on a hydrogen spark gap under hundreds of atmospheres pressure, and the multi-switch design uses the physical length of part of the pulse line to set the pulse length. These determine the lower frequency performance.

The high gain non-dispersive antenna approach is embodied at present in the Impulse Radiating Antenna (IRA) ." This system has a hydrogen spark gap at 1500 psi located at the focus of a large $(2 \mathrm{~m})$ dish with four feed arms that properly terminate the pulser to the edges of the dish in the characteristic impedance of the system. The system operates at a relatively modest voltage at present, $+/-70 \mathrm{kV}$. Pulser needs for this technology are for higher voltage compact pulsers to drive the antenna from the focal point, or for schemes like the half-IRA concept that could use a ground plane with an $\mathrm{H}$-series pulser or other pulser integrated into it.

The photoconductive semiconductor switched (PCSS) array approach has been embodied in the GEM series of pulsers to date. ${ }^{12}$ Tremendous improvements in the switched voltage, lifetime of the switches, pulsed power used to charge the switched lines, and control system have led to the successful demonstration of the PCSS systems. The Phillips Laboratory is pursuing further improvements to the technology with a view of increasing the voltage delivered to each radiating element. The effort is proceeding along two complementary lines. First, the photoconductive switches are being improved in an effort that is joint between the Phillips Laboratory and the Army' Research Laboratory at Ft. Monmouth. The Army Research Laboratory is providing inproved Ga As photoconductive switches, with the goal of extending to higher switched voltage devices that also have increased lifetime. Better contact technology and improved switch geometry are also part of the effort. The second line of attack on the problem seeks to apply a pulsed power concept developed initially for bremsstrahlung and narrowband modulator purposes. Jointly with the University of Texas at Dallas Center for Quantum Electrodynamiss, we are pursuing the stacked blumlein technology. This approach allows for the voltage switched to be multiplied by the number if blumleins stacked in series at 
the output. ${ }^{13}$ The work at UTD has in this year converted from thyratron switching used previously to photoconductive switching. An important feature to having this technology work is having a reliable means for limiting both the energy delivered to the switched line and for limiting the time the switch sees the voltage. At present the UTD laboratory arrangement for this is a single blumlein pulser (Figure 5) that provides a voltage that can be varied up to 100 $\mathrm{kV}$, has a pulse that rises in $80 \mathrm{~ns}$, falls in $80 \mathrm{~ns}$, and has an 80 ns FWHM and a maximum PRF of $1 \mathrm{kHz} .{ }^{14}$

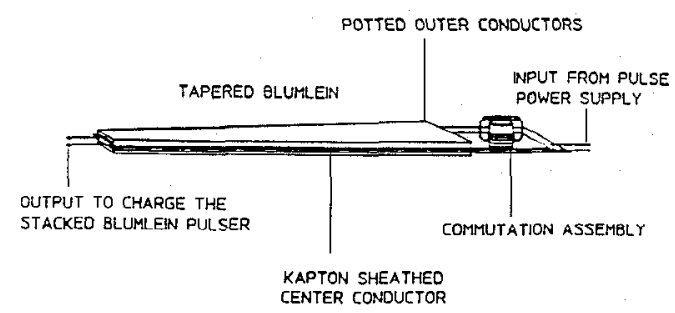

Figure 5. Schematic Drawing of Charging Pulse Compression Module Used to Pulse Charge Stacked Blumtein UWB Pulsers.

The rather bulky pulser provides more energy than is needed to charge a single line, and a series limiting resistor is used in the charging circuit to compensate for this. A better solution would be a compact, reliable and inexpensive means to charge the pulsed lines switched by the photoconductive switches. Ideally it would have a rise time of $5-10 \mathrm{~ns}$, a flat pulse length of $20-30 \mathrm{~ns}$, and provide about $200 \mathrm{~mJ}$ to each blumlein. It would be useful to have available a variable repetition rate up to $10 \mathrm{kHz}$.

These represent a snapshot of the currently perceived needs for power modulators for high power microwaves. Further advances in practical, compact, lightweight pulsed power will be needed to get this technology out of the laboratory and into practical use. Significant possibilities open up if this can be achieved.

\section{References}

1. G. Huttlin, M. Bushell, D. Conrad, D. Davis, K. Ebersole, D. Judy, P. Lezcano, M. Litz, N. Pereira, B. Ruth, D. Weidenheimer, and F. J. Agee, "The Reflex-Diode HPM Source on Aurora," IEEE Transactions on Plasma Science 18, 618-625 (1990).
2. M. Friedman et al., J. Appl. Phys. 64, 3353 (1988); Rev. Sci. Instrum. 61, 171 (1990); IEEE Transactions on Plasma Science 18, 553 (1990); Proc. of the SPIE 1872, 2 (1993); Phys. Rev. Lett. 74, 322 (1995), 75, 1214 (1995), 50, 1922 (1983).

3. K. J. Hendricks et al, "The Extraction of RF Power from an Injection Locked Relativistic Klystron Oscillator," Phys. Rev. Lett. 76, 154 (1996).

4. S. E. Calico, M. C. Clark, R. W. Lemke, and M. C. Scott, "Experimental and Theoretical Investigations of a Magnetically Insulated Line Oscillator (MILO), Proc. SPIE 2557, 50 (1995). 5. M. V. Fazio, W. B. Haynes, B. E. Carlsten, and R. M. Stringfield, IEEE Trans. on Plasma Sci. 22, (5), 740 (1994).

6. R. B. Miller, C. A. Muehlenweg, K. W. Habiger, and J. R. Clifford, IEEE Trans. Plasma Sci. 22, (5), 701 (1994).

7. R. J. Barker and F. J. Agee, Proc. SPIE 2557, 300 (1995).

8. J. Benford, F. J. Agee, R. J. Barker, et al, AFOSR topical meeting on pulse shortening, Int. Conf. on Plasma Sci. (1995).

9. K. J. Hendricks and S. E. Calico, private communication.

10. S. E. Calico and M. C. Scott, "Performance Enhancement of the Phillips Laboratory RepRate Pulser with a Triggered Output Switch," Conference Record of the 1994 Twenty-First International Power Modulator Symposium, 5659 (1994).

11. S. E. Calico, F. J. Calico, M. C. Clark, R. M. Lemke, and M. C. Scott, "Rep-Rate Operation of a Magnetically Insulated Line Oscillator," Proc. of the First International Workshop on Crossed Field Devices, Ann Arbor, Ml, 147-158 (1995). 12. F. J. Agee, D. W. Scholfield, W. Prather, and J.W. Burger, "Powerful Ultra-Wide Band Emitters: Status and Challenges," Proc. SPIE 2557, 98-106 (1995).

13. F. Davanloo, D. L. Borovina, C. B. Collins, F. J. Agee, and L. E. Lingsley, "Repetitively Pulsed High Power Stacked Blumlein Generators," Nucl. Instr. and Methods B99, 713-716 (1995).

14. D. L. Borovina, R. K. Krause, F. Davanloo, C. B. Collins, "Switching the Stacked Blumlein Pulsers: Status and Issues," in Proceedings of the 10th IEEE International Pulsed Power Conference, July 10-13, 1995, Albuquerque, NM (to be published). 